\title{
Predictors of Mortality in Primary Surgical Repair of Postinfarction Ventricular Septal Defects: The Leipzig Experience
}

\author{
Maximilian Vondran, MD, ${ }^{1,2}$ Jens Garbade, MD, PhD,${ }^{1}$ Sven Lehmann, MD, PhD,${ }^{1}$ Johannes Laessing, $, 1,3$ \\ Mahmoud S. Wehbe, MD, ${ }^{1,4}$ Ardawan J. Rastan, MD, PhD,${ }^{2}$ Michael A. Borger, $\mathrm{MD}, \mathrm{PhD},{ }^{1}$ \\ Thomas Schroeter, MD ${ }^{1}$ \\ ${ }^{1}$ University Department for Cardiac Surgery, Heart Center Leipzig, Leipzig, Germany \\ ${ }^{2}$ Department of Cardiac and Vascular Thoracic Surgery, Philipps-University Hospital Marburg, Marburg, Germany \\ ${ }^{3}$ Institute of Sports Medicine \& Prevention, Faculty of Medicine, University of Leipzig, Leipzig, Germany \\ ${ }^{4}$ Department of Cardiac Surgery, Schuechtermann-Klinik, Bad Rothenfelde, Germany
}

\section{ABSTRACT}

Background: A postinfarction ventricular septal defect (pVSD) as a complication of acute myocardial infarction (AMI) is associated with high mortality. This retrospective, single-center study aimed to identify predictors of early and long-term outcomes in patients undergoing primary surgical repair of pVSD managed by various surgical techniques.

Methods: We reviewed data from 77 consecutive patients who underwent primary surgical repair after pVSD in our institution. Prospectively collected demographic and perioperative data were analyzed retrospectively. Predictors of 30-day mortality and long-term outcome were assessed.

Results: pVSD was anterior in 45 patients $(58.4 \%)$ and posterior in $32(41.6 \%)$. Buttressed mattress suture $(\mathrm{n}=9$, $11.7 \%)$, simple single septal patch $(\mathrm{n}=34,44.2 \%)$, simple double septal patch $(\mathrm{n}=2,2.6 \%)$, sandwich double patch $(\mathrm{n}=$ $1,1.3 \%)$, and the infarct exclusion technique $(\mathrm{n}=31,40.3 \%)$ were performed for surgical closure. Fifty-three patients (68.8\%) had preoperative cardiogenic shock. The 30-day mortality was $42.8 \%$ (33 patients). Independent risk factors of 30-day mortality were duration between AMI and surgery $<7$ days (odds ratio $[\mathrm{OR}] 5.229, \mathrm{P}=.011$ ), preoperative absence of diuretics (OR 6.913, $\mathrm{P}=.005)$, and preoperative cardiogenic shock (OR 3.558, $\mathrm{P}=.011)$. Cumulative survival rates at 1,5 , and 10 years were $57.1 \%, 57.1 \%$, and $31.2 \%$, respectively.

Conclusion: In pVSD, the 30-day mortality remains high, and preoperative cardiogenic shock significantly influenced mortality in our study. None of the surgical techniques or materials used in our investigation influenced the outcome.

\section{INTRODUCTION}

Acute myocardial infarction (AMI) remains one of the leading causes of death in developed countries. However, thanks to increasing progress in treatment options, its mortality rate has fallen significantly over the past 2 decades [Statistisches Bundesamt 2015]. The mechanical complications of AMI such as postinfarction ventricular septal defect (pVSD) occur seldom $(0.17 \%$ to $0.34 \%)$ but are soon fatal with medical therapy only [Coskun 2009; Crenshaw 2000; Lee 1962; Moreyra 2010]. Notwithstanding the milestones in the gold-standard surgical treatment of pVSD [David 1995], 30-day mortality among these patients remains high: namely, $36.0 \%$ to $42.9 \%$ in recent studies [Arnaoutakis 2012; Lundblad 2014; Papadopoulos 2009; Takahashi 2015]. Not even the introduction of percutaneous, catheter-based closure of the pVSD has resulted in a real revolution, since the mortality rates for this procedure are similar and this method can currently only be used for small to medium-sized pVSDs [Schlotter 2016]. In addition, various risk factors are known to be responsible for a poor perioperative outcome, including cardiogenic shock (CS), emergency surgery, early repair, right ventricular dysfunction, posterior $\mathrm{pVSD}$, renal impairment, and complex pVSD [Cinq-Mars 2016].

The different surgical approaches and the extent of the operation have regained renewed interest in recent years with the increasing use of percutaneous pVSD closure. The aim of this retrospective study was to determine the influence of different surgical methods, the extent of surgery, and the use of different materials on 30-day mortality. We also explored independent risk factors for 30-day mortality and long-term outcome to further optimize the perioperative outcome of these patients.

\section{METHODS}

\section{Patient Cobort and Characteristics}

At our institution, 77 patients underwent surgical closure for pVSD between October 1994 and January 2016. We only included patients in our study with a new diagnosis 
of ventricular septal defect (VSD) due to myocardial infarction based on findings from right heart catheterization and/or transthoracic/transesophageal echocardiography. If a surgical or percutaneous interventional or surgical VSD occlusion had been attempted previously, the patient was excluded. We performed a retrospective analysis of the computerized hospital databases and the patient health records, considering demographics; pre-, intra-, and postoperative data; and survival at 30 days and the longest follow-up time point available.

\section{Definition of CS}

Cardiogenic shock was defined as persistent systolic blood pressure $\leq 90 \mathrm{mmHg}$ or vasopressors required to maintain blood pressure $\geq 90 \mathrm{mmHg}$; evidence of end-organ failure (eg, urine output $\leq 30 \mathrm{ml} / \mathrm{h}$, cold skin and extremities, serum lactate $\geq 2 \mathrm{mmol} / \mathrm{l}$ ); evidence of elevated left ventricular filling pressures with clinical signs of pulmonary congestion; and/ or the use of mechanical circulatory support devices (MCS).

\section{Operative Procedures}

All procedures were performed using a standard median sternotomy. The occlusion of the pVSD was performed using various techniques during cardiopulmonary bypass. A buttressed mattress suture without a patch was used in 9 patients (11.7\%); a simple single-patch, simple double-patch, or sandwich double-patch was established in $34(44.2 \%)$, $2(2.6 \%)$, and $1(1.3 \%)$, respectively. The infarct exclusion technique according to David et al [1995] was used in 41 $(40.3 \%)$ patients, and 51 patients $(66.2 \%)$ needed concomitant surgery.

\section{Statistical Methods}

Unless otherwise indicated, categorical variables are presented as numbers and percentages, and continuous parameters are expressed as mean \pm standard deviation (SD). Fisher's exact or $\chi^{2}$ test was used to assess differences for categorical variables, and Student's t test or the Wilcoxon rank-sum test was applied for continuous parameters, as appropriate. Allcause survival rates were analyzed by using the method of Kaplan-Meier. The log-rank test calculated statistical differences. Patients were divided into those who survived or did not survive 30 days after surgery for risk assessment. Multivariate analysis was performed using a logistic regression model to differentiate independent risk factors for 30-day all-cause mortality and Cox regression model for total allcause mortality.

All tests were conducted 2 -sided at the significance level $\alpha$ $=0.05$. Unless otherwise stated, the analyses were performed using IBM SPSS Statistics for Macintosh, version 26.0 (IBM Corp., Armonk, NY).

\section{Ethical Concerns}

All authors had full access to datasets and maintained data integrity. They read and agree to the final paper as written. The local Ethics Committee approved the study. The study design, pseudonymous data acquisition, and data publication adhered to the Declaration of Helsinki.

\section{RESULTS}

Preoperative patient characteristics are summarized in Table 1 for all patients and according to 30-day survival. The mean age of the entire cohort was $68.7 \pm 9.4$ years. Left ventricular ejection fraction was $44.0 \% \pm 15.0 \%$, and the logistic European System for Cardiac Operative Risk Evaluation (EuroSCORE) was $42.4 \% \pm 25.8 \%$. Fifty-three patients $(68.8 \%)$ presented in CS preoperatively and were implanted with an MCS; 36 patients (46.8\%) received an intra-aortic balloon pump, and 4 (5.2\%) extracorporeal life support (ECLS) preoperatively. The group of nonsurvivors was older $(71.7 \pm 8.8$ versus $66.4 \pm 9.3$ years, $P=.014)$ and had a shorter time of hospital admission to surgery $(4.8 \pm$ 7.6 versus $9.8 \pm 9.4$ days, $P=.014$ ) as well as of AMI occurrence to surgery $(11.1 \pm 14.9$ versus $32.0 \pm 39.2$ days, $P=$ $.007)$. In addition, the nonsurvivor group was treated more frequently by emergency surgery $(31.2 \%$ versus $18.2 \%, P$ $=.001)$ and was more often in CS preoperatively $(39.0 \%$ versus $29.9 \%, P=.001$ ).

Periprocedural details are shown in Table 2. There were no differences between the group of nonsurvivors and survivors concerning surgical technique, extent of the procedure, or patch material used, but the group of nonsurvivors had a longer heart-lung machine time than the group of survivors $(128.4 \pm 52.6$ versus $101.6 \pm 49.6 \mathrm{~min}, P=.028)$. The infarct exclusion technique described by David et al [1995] had no impact on 30-day mortality in our cohort $(P=.485)$. There was a tendency for surgical treatment of ventricular aneurysm to be more frequent $(23.4 \%$ versus $9.1 \%, P=.087)$, access to $\mathrm{pVSD}$ occlusion to be via the left ventricle $(33.8 \%$ versus $23.4 \%, P=.817)$, and incomplete revascularization to be less frequent $(18.2 \%$ versus $22.1 \%$, $P=.103)$ in the group of survivors, but these differences were not significant.

Postoperative outcomes are summarized in Table 3. After surgery, 20 patients $(26.0 \%)$ received a new or additional MCS. However, the nonsurvivor group had more frequent postoperative myocardial infarction $(7.8 \%$ versus $1.3 \%, P=$ $.038)$, need for resuscitation $(10.7 \%$ versus $0.0 \%, P=.001)$, and requirement for dialysis $(19.5 \%$ versus $10.4 \%, P=.013$ ) compared with the survivor group. Furthermore, 6 patients $(7.8 \%)$ had residual VSD after surgery and $13(16.9 \%)$ at discharge. Because of a large persistent shunt, 9 patients $(11.7 \%)$ had to undergo redo surgery; 4 of 5 of these patients who needed a reoperation within 30 days died. The other 4 patients, who all had $>1$ month between initial surgery and redo surgery, survived the reoperation.

The all-cause 30-day mortality of the population was $42.8 \%$ (33 patients). Figure 1A shows the Kaplan-Meier curve of the entire cohort, and Figure $1 \mathrm{~B}$ illustrates the 30 -day mortality of the patients with and without CS. The most common cause of death during the first 30 days after surgery was low cardiac output syndrome (20 patients; $26.0 \%)$. However, only 4 patients (5.2\%) died of multiorgan failure, and 1 patient $(1.3 \%)$ died of a neurological complication. In 10 other patients $(13.0 \%)$, the ultimate cause of death could not be reconstructed reliably from the available 
Table 1. Preoperative Patient Characteristics*

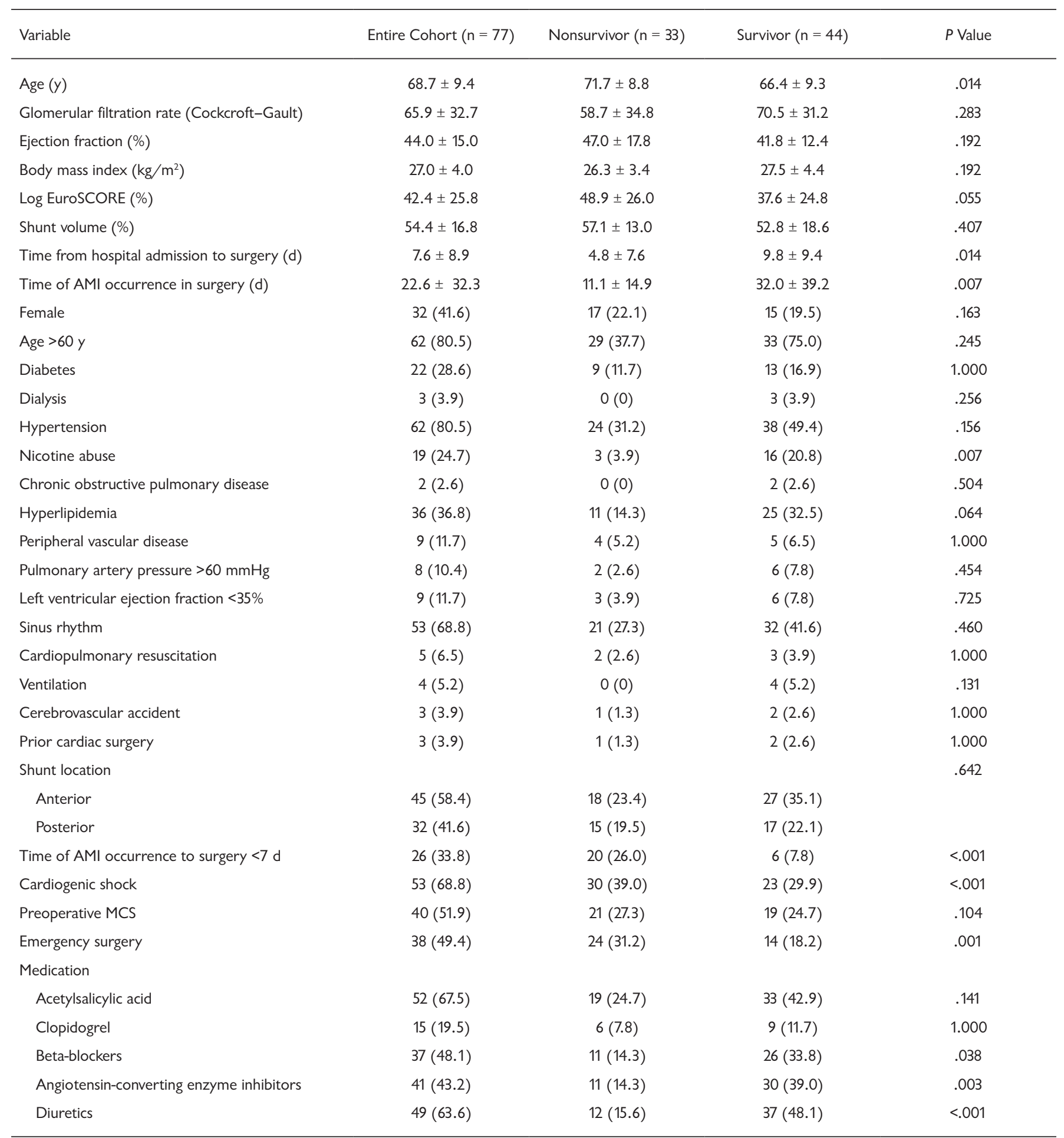

*Categorical variables are presented as $\mathrm{n}(\%)$ and continuous parameters as mean \pm SD. P values are for comparison of survivors and nonsurvivors (30-day mortality). 
documentation. The cumulative 1-, 5-, and 10-year survival rates were $57.1 \%, 57.1 \%$, and $31.2 \%$, respectively (Figure 1A). The follow-up period for the entire cohort was $3.2 \pm 3.9$ years. Patients who survived $>30$ days were followed for 5.6 \pm 4.9 years.

Multiple logistic regression analysis revealed that a duration between AMI and surgery of $<7$ days (odds ratio [OR]
5.229; $P=.011$ ), preoperative absence of diuretics (OR 6.913; $P=.005)$, and preoperative CS (OR 3.558; $P=.040)$ were independent predictors of 30-day mortality (Table 4 ). The only independent predictor of overall mortality was cardiopulmonary bypass time (hazard ratio 1.013, confidence interval $[\mathrm{CI}] 1.004$ to $1.021 ; P=.002)$, as determined by Cox regression analysis.

Table 2. Periprocedural Details*

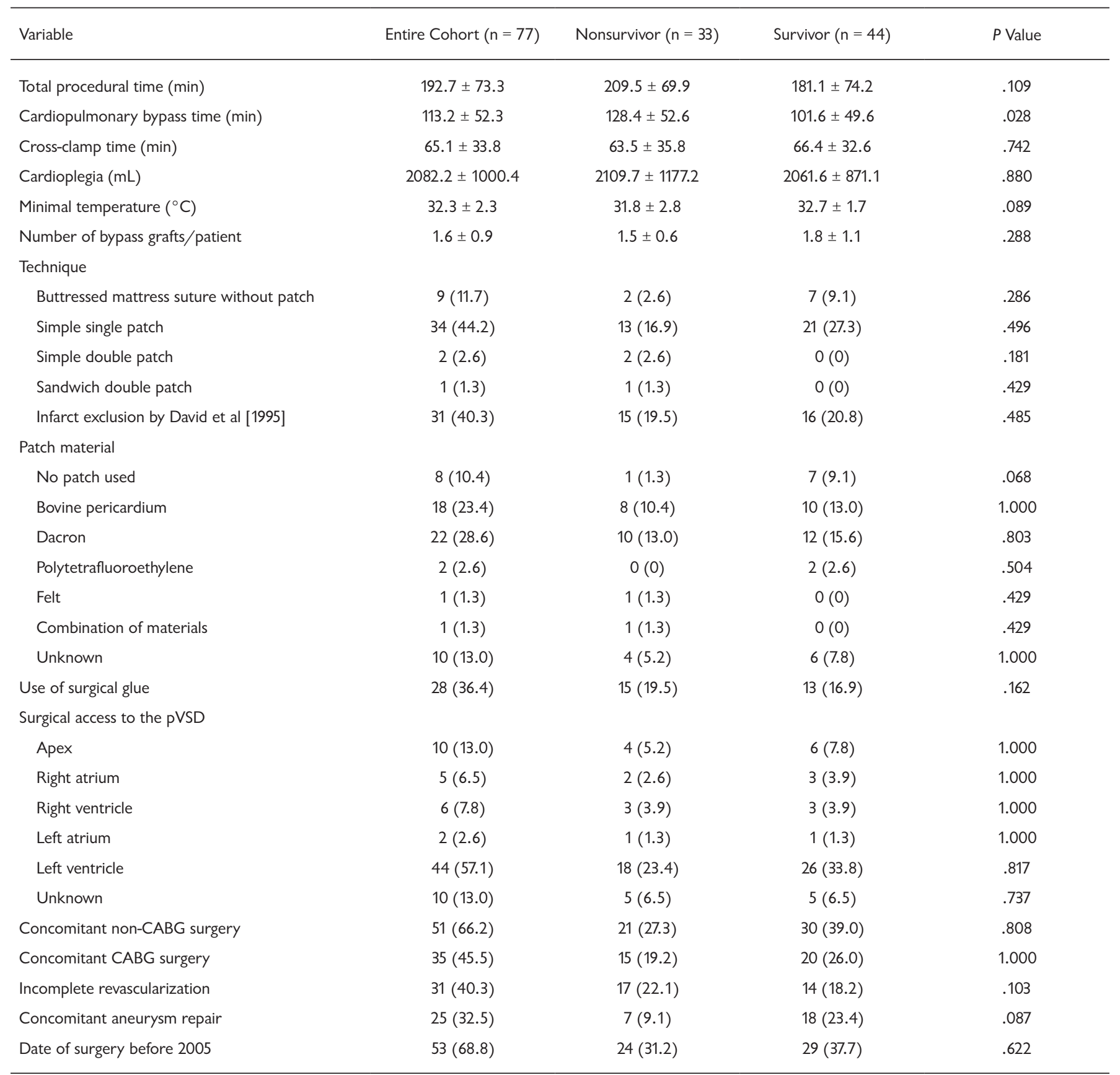

*Categorical variables are presented as $\mathrm{n}(\%)$ and continuous parameters as mean \pm SD. P values are for comparison of survivors and nonsurvivors (30-day mortality). CABG indicates coronary artery bypass graft surgery. 
Table 3. Postoperative Outcomes*

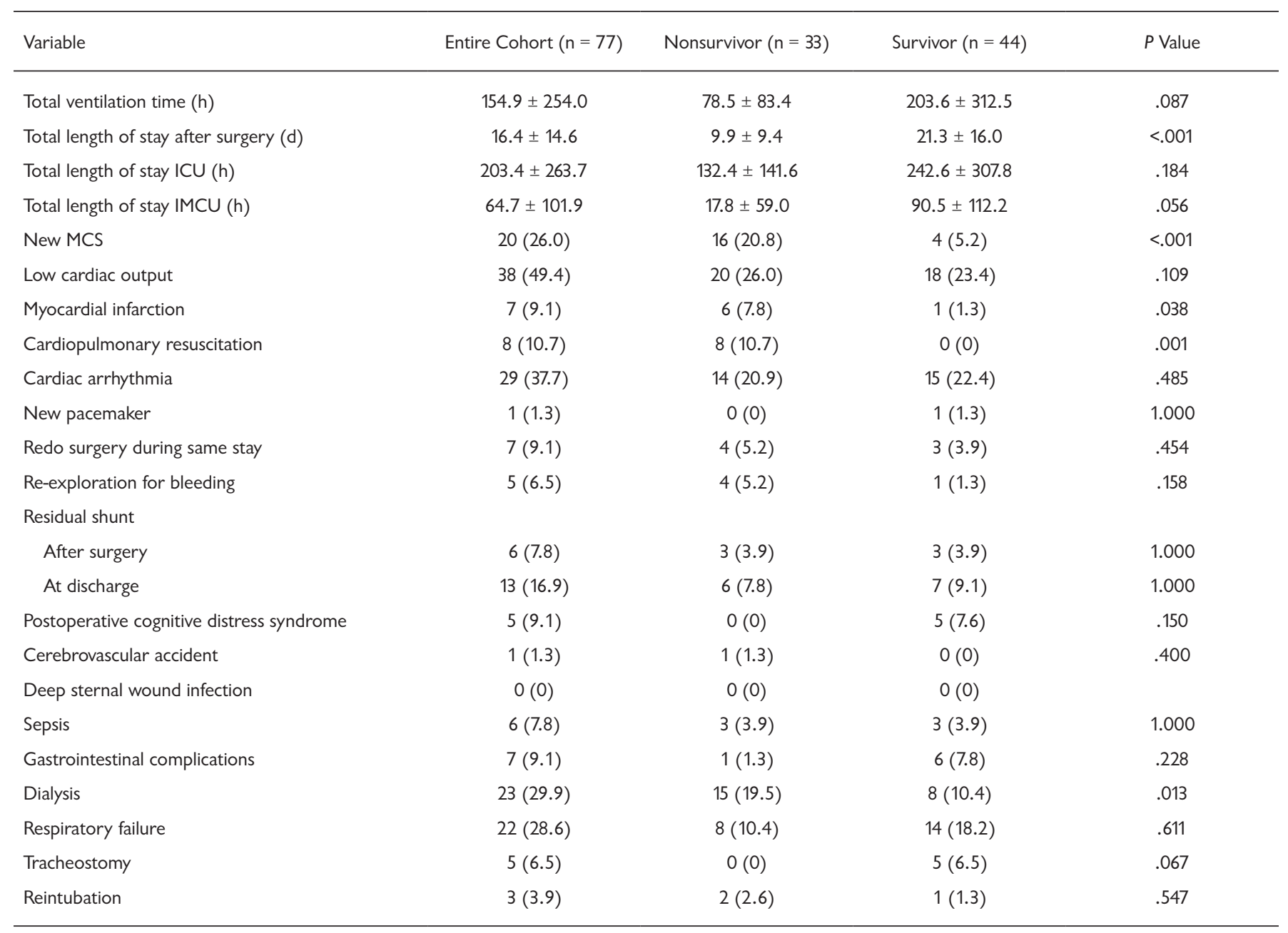

*Categorical variables are presented as $\mathrm{n}(\%)$ and continuous parameters as mean \pm SD. P values are for comparison of survivors and nonsurvivors (30-day mortality). ICU indicates intensive care unit; IMCU, intermediate care unit.
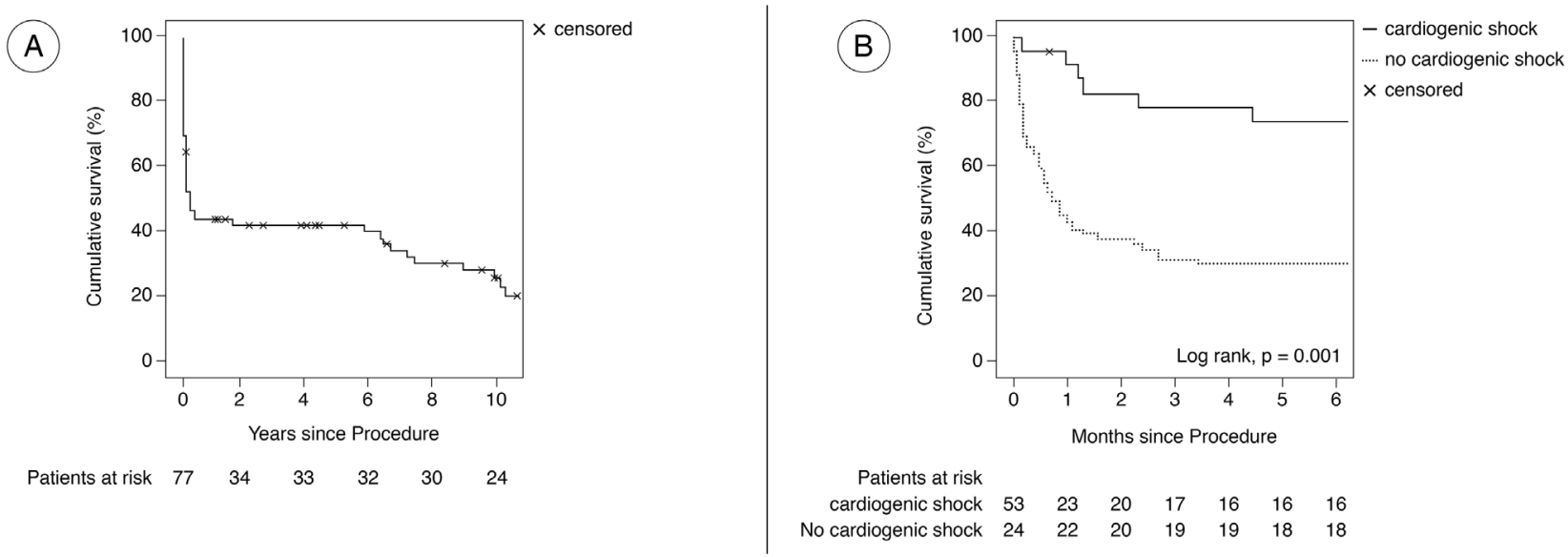

Figure 1. Kaplan-Meier survival curves for all-cause mortality of the entire cohort (A) and comparison between patients with and without CS (B). 
DISCUSSION

In this study, we investigated the risk factors for all-cause 30-day and overall mortality in patients with pVSD who underwent primary surgical repair via various techniques at our institution. The 30-day mortality rate of these patients was $42.8 \%$; independent predictors were duration between AMI and surgery $<7$ days, preoperative absence of diuretics, and preoperative CS. Our detailed investigation of the surgical technique of pVSD closure and of concomitant surgeries revealed no variable that significantly influenced mortality. Longer cardiopulmonary bypass time proved to be an independent risk factor for overall all-cause mortality.

\section{Perioperative Mortality and Risk Factors}

Our cohort's $42.8 \% 30$-day mortality rate is relatively high, but is comparable to outcomes of the 2 benchmark studies on this issue. The Global Utilization of Streptokinase and t-PA for Occluded Coronary Arteries (GUSTO) trial's operative mortality rate was $47 \%$ [Crenshaw 2000], and it was $42.9 \%$ in the study by Arnaoutakis et al [2012], which used the Society of Thoracic Surgeons National Database and was published a little over a decade after the GUSTO trial. In recent studies with smaller cohorts, however, perioperative mortality ranges from at least $4.3 \%$ [Kim 2015] to a maximum of $65.0 \%$ [Cinq-Mars 2016], with the majority of studies ranging between 20\% and 40\% [Lundblad 2014; Okamoto 2016; Papadopoulos 2009; Takahashi 2015; Yam 2013].

In patients with pVSD, preoperative CS appears to be the most important variable among risk factors for in-hospital mortality, and numerous authors have substantiated this link over the years [Arnaoutakis 2012; Cinq-Mars 2016; Coskun 2009; Pang 2013; Papadopoulos 2009]. CS was also an independent variable for 30-day mortality in our patient population. Note that the various studies applied no uniform definition of CS, and CS was sometimes not defined at all. However, it seems logical that forced emergency or ultima ratio surgery would be associated with a poor outcome because of failing hemodynamics. In this context, the risk factor present at the time of pVSD surgery is always reported. A brief interval between AMI and surgery usually results in poor in-hospital/30-day mortality rates [Cinq-Mars 2016; Lundblad 2014; Papadopoulos 2009; Takahashi 2015], which is consistent with our finding that an interval of $<7$ days between AMI and surgery is an independent risk factor for 30-day mortality. In our opinion, patients need to be hemodynamically stabilized before undergoing surgery, as several authors have shown or recommended [Arnaoutakis 2012; Cinq-Mars 2016; Takahashi 2015; Trivedi 2015], so that the infarct-damaged tissue can scar as much as possible before surgery. Hemodynamic stabilization should be attempted via ECLS if necessary, and the culprit lesion should be immediately revascularized percutaneously. In this context, bridging therapy with a percutaneous septal occluder device should also be considered until the patient has been stabilized hemodynamically and can be admitted for surgery.

Interestingly, the absence of diuretic use upon hospital admission was also an independent predictor of 30-day mortality. Almost 70\% of our patients were in CS before surgery; therefore, we consider it logical that they were no longer on beta-blockers or angiotensin-converting enzyme inhibitors, as they were usually catecholamine dependent. However, it seems equally plausible that optimal diuretic therapy could play a decisive role in patients' short-term outcomes. By optimizing the preload, pulmonary and local edema can be reduced or prevented, and an additional volume load on the right ventricle can be minimized. In addition, congestion of the liver and thus potential liver cell death can be prevented. There is little data available about the influence of preoperative medication in pVSD patients undergoing cardiac surgery. A study by Arnaoutakis et al [2012] investigated only beta-blockers and fat-lowering drugs: with about $50 \%$ of the patients in CS, significantly more patients who survived the first 30 days had been taking a beta-blocker. In a study by Thiele et al [2009], there was no difference in survival for those on beta-blockers. In this interventionally treated group, $55 \%$ of patients were in CS. We are not aware of any other data regarding preoperative diuretic therapy in patients with pVSD and subsequent surgical occlusion.

Impact of Surgical Repair Technique on Perioperative Mortality

The surgical technique for closing a pVSD has evolved over the past half-century, and perioperative outcomes have improved as a result of several milestones, from the first description of the technique by Cooley et al [1957] to a further iteration by Daggett et al [1977] to the infarct exclusion technique according to David et al [1995]. Although there are no randomized, controlled studies available that compare the different methods, the David infarct exclusion technique is still regarded as the preferred technique. In a metadata study by Lundblad and Abdelnoor [2014], a 30-day mortality rate of $16.7 \%$ was demonstrated with infarct exclusion in 42 patients. Thus, this pVSD closure technique was superior to that of Daggett et al [1977] for both early (30 days) and late survival.

Table 4. Results of the Logistic Regression Analysis for Independent Predictors of 30-Day Mortality

\begin{tabular}{llll}
\hline Variable & OR & $95 \% \mathrm{Cl}$ & $P$ Value \\
\hline Time of AMI occurrence to surgery $<7 \mathrm{~d}$ & 5.229 & 1.451 to 18.847 & .011 \\
Preoperative CS & 3.558 & 1.058 to 11.967 & .040 \\
Preoperative absence of diuretics & 6.913 & 1.808 to 26.430 & .005 \\
Posterior pVSD & 2.755 & 0.759 to 9.996 & .123
\end{tabular}


It should be noted that the 2 groups in this study fall into different periods, as mentioned by Lundblad and Abdelnoor [2014]: the study covers 30 years, with the method of Daggett et al [1977] mainly used in the first half and the technique of David et al [1995] in the second half. In this study, the improvement of general perioperative management may also play a role that should not be underestimated. In addition, it is mentioned that the same surgeons who were initially trained with the method of Daggett et al [1977] in the first half of the study also used the technique of David et al [1995] later, after achieving a certain level of expertise, and then preferred it to the Daggett method. In the collective data first described by David et al [1995], a perioperative mortality rate of $13.6 \%$ was reported; this was similar to the 30-day mortality rate of $16.7 \%$ reported by Lafci et al [2006] for patients treated with the David infarct exclusion technique. Unfortunately, these values were not upheld in our collective: the 41 patients treated by the infarct exclusion technique had a 30-day mortality rate of $36.6 \%$. However, our results are similar to those described in the work of Papadopoulos et al [2009]. In their group of 32 patients who underwent consecutive pVSD surgery using the infarct exclusion technique, the 30-day mortality rate was $31.5 \%$.

Several groups have explored modifications of the technique of David et al [1995]. A recent paper by Kim et al [2015] presented results of the use of a slight but not uniformly modified infarct exclusion technique. They operated on 17 of their 23 patients with the modified technique. The defect was excluded using either a double patch with a conically shaped patch or a triple patch without a conically shaped patch. The remaining ventricular cavities excluded from the patch were then filled with a sealant of purified bovine serum albumin and glutaraldehyde. The 30-day mortality rate of these patients was $4.3 \%$. Another modification of the infarct exclusion is the triple-patch technique, according to Okamoto et al [2016], in which the remaining spaces between the patches are also filled with surgical glue. In that study, a 30 -day mortality rate of $23.8 \%$ was reported for a cohort of 21 consecutive patients. In addition to patch configurations, most new or modified infarction exclusion techniques explicitly emphasize using surgical adhesive to fill the ventricular cavities remaining after patch application [Isoda 2012; Kim 2015; Okamoto 2016]. We also applied surgical adhesive to just over a third of our patients, but its use did not affect 30-day mortality. However, in both the Kim et al [2015] and Okamoto et al [2016] studies, $>80 \%$ of the patients had an anterior defect. It is described in the literature that patients with a posterior pVSD have a worse outcome [Coskun 2009; Cummings 1988; Sibal 2010]. In our cohort, with an approximately balanced distribution of the respective groups (anterior pVSD 58.4\% versus posterior pVSD $41.6 \%$ ), we did not observe any survival disadvantage for patients with posterior pVSD.

\section{Interventional pVSD Closure and Potential Hybrid Concepts}

It is also possible to close a pVSD using catheter-based intervention. Since its introduction by Lock et al [1988], this procedure, as with surgical pVSD closure, has been developed further so that the all-cause 30-day mortality rate has fallen to $14 \%$ to $66 \%$ [Holzer 2004; Maltais 2009; Thiele 2009; Zhu 2013]. However, there are still some limitations to the use of percutaneous pVSD closure due to patient anatomy and the configuration and size of the available occluders [Holzer 2004; Schlotter 2016; Thiele 2009; Zhu 2013]. In our opinion, a hybrid concept for the closure of a pVSD may offer an opportunity to reduce mortality rates further. Different kinds of hybrid approaches have been described [Gulkarov 2010; Jorge 2012; Vondran 2016]. The largest known case series contains 6 successful hybrid treatments. These patients had acute heart failure or CS and had a sizeable residual left-toright shunt. The patients were hemodynamically stabilized before surgery, and only 1 patient (17\%) died after reoperation due to occluder dislocation caused by endocarditis [Vondran 2016].

In the acute phase of pVSD therapy shortly after AMI, the focus should be on hemodynamic stabilization and shunt reduction. All currently available data suggest that CS is the critical variable for short-term survival [Arnaoutakis 2012; Cinq-Mars 2016; Coskun 2009; Pang 2013; Papadopoulos 2009]. The time between AMI and the associated fragility of the myocardium are also essential factors. If the patient has survived the acute phase, they have excellent chances of surviving in the long term [Arnaoutakis 2012; Cinq-Mars 2016; Takahashi 2015]. We think a small, residual shunt may play a subordinate role in in-hospital mortality, but data supporting this suggestion are lacking. Patients without CS should ideally wait until scarring of the pVSD has occurred before undergoing the final surgery. Depending on the character of the pVSD, implantation of a percutaneous occluder could be attempted beforehand for shunt reduction to counteract hemodynamic deterioration. A residual shunt should always be reinterpreted in a symptom-free interval before hemodynamic deterioration. For this reason, we recommend that the modern interventional heart surgeon also learn this technique to be able to offer the best possible therapy.

\section{Limitations}

Our study has all the limitations of a single-center retrospective study. Because of the rarity of pVSD, the number of patients in our cohort is small, and only small subgroups could be established to calculate independent predictors of all-cause 30-day and overall mortality.

\section{Conclusions}

In pVSD, the all-cause 30-day mortality rate remains high, and preoperative CS still influences mortality significantly. In our study, the outcome was not significantly influenced by surgical technique or the material applied. However, a preoperatively implanted MCS as a bridge to definitive surgical therapy may improve the outcome in patients with CS. A good cooperation between interventional cardiologists and cardiac surgeons in the heart team should be strived for to optimally treat patients with pVSD using a hybrid approach. Further studies are needed to explore this proposed approach. 
ACKNOWLEDGMENTS

The authors thank Bettina Hentschel (Center for Clinical Trials, University of Leipzig, Leipzig, Germany) for the statistical support in this work. The authors thank Peter Meyer (Bureau $\mathrm{M} / \mathrm{M}$, Stuttgart-Leipzig, Germany) for preparing the figures.

\section{REFERENCES}

Arnaoutakis GJ, Zhao Y, George TJ, Sciortino CM, McCarthy PM, Conte JV. Surgical repair of ventricular septal defect after myocardial infarction: Outcomes from the society of thoracic surgeons national database. Ann Thorac Surg 2012;94:436-443.

Cinq-Mars A, Voisine P, Dagenais F et al. Risk factors of mortality after surgical correction of ventricular septal defect following myocardial infarction: Retrospective analysis and review of the literature. Int J Cardiol 2016;206:27-36.

Cooley DA, Belmonte BA, Zeis L, Schnur S. Surgical repair of ruptured interventricular septum following acute myocardial infarction. Surgery 1957;41:930-937.

Coskun KO, Coskun ST, Popov AF, et al. Experiences with surgical treatment of ventricle septal defect as a post infarction complication. J Cardiothorac Surg 2009; $4: 3$

Crenshaw BS, Granger CB, Birnbaum Y et al. Risk factors, angiographic patterns, and outcomes in patients with ventricular septal defect complicating acute myocardial infarction. Gusto-i (Global Utilization of Streptokinase and TPA for Occluded Coronary Arteries) trial investigators. Circulation 2000;101:27-32.

Cummings RG, Reimer KA, Califf R, Hackel D, Boswick J, Lowe JE. Quantitative analysis of right and left ventricular infarction in the presence of postinfarction ventricular septal defect. Circulation 1988;77:33-42.

Daggett WM, Guyton RA, Mundth ED et al. Surgery for post-myocardial infarct ventricular septal defect. Ann Surg 1977;186:260.

David TE, Dale L, Sun Z. Postinfarction ventricular septal rupture: Repair by endocardial patch with infarct exclusion. J Thorac Cardiovasc Surg 1995;110:1315-1322.

Gulkarov IM, Anez-Bustillos L, Wong SC, Salemi A. A hybrid approach in the treatment of post-myocardial infarction ventricular septal defect. Cardiology 2010;116:183-185.

Holzer R, Balzer D, Amin Z et al. Transcatheter closure of postinfarction ventricular septal defects using the new Amplatzer muscular VSD occluder: Results of a U.S. Registry. Catheter Cardiovasc Interv 2004;61:196-201.

Isoda S, Osako M, Kimura T et al. Midterm results of the 'sandwich technique' via a right ventricle incision to repair post-infarction ventricular septal defect. Ann Thorac Cardiovasc Surg 2012;18:318-321.

Jorge C, de Oliveira EI, Martins SR, Nobre A, da Silva PC, Diogo AN. Hybrid closure of postinfarction ventricular septal rupture enlargement after transcathether closure with amplatzer occluder. Eur Heart J Acute Cardiovasc Care 2012;1:57-59.

Kim IS, Lee JH, Lee DS et al. Surgical outcomes of a modified infarct exclusion technique for post-infarction ventricular septal defects. Korean J Thorac Cardiovasc Surg 2015;48:381-386.

Lafci B, Yakut N, Goktogan T et al. Repair of post-infarct ventricular septal rupture with an infarct-exclusion technique: Early results. Heart Surg Forum 2006;9:E737-E740.
Lee WY, Cardon L, Slodki SJ. Perforation of infarcted interventricular septum: Report of a case with prolonged survival, diagnosed ante mortem by cardiac catheterization, and review of the literature. Arch Intern Med 1962;109:731-741.

Lock JE, Block PC, McKay RG, Baim DS, Keane JF. Transcatheter closure of ventricular septal defects. Circulation 1988;78:361-368.

Lundblad R, Abdelnoor M. Surgery of postinfarction ventricular septal rupture: The effect of David infarct exclusion versus Daggett direct septal closure on early and late outcomes. J Thorac Cardiovasc Surg 2014;148:2736-2742.

Maltais S, Ibrahim R, Basmadjian AJ, et al. Postinfarction ventricular septal defects: Towards a new treatment algorithm? Ann Thorac Surg 2009;87:687-692.

Moreyra AE, Huang MS, Wilson AC, et al. Trends in incidence and mortality rates of ventricular septal rupture during acute myocardial infarction. Am J Cardiol 2010;106:1095-1100.

Okamoto Y, Yamamoto K, Asami F et al. Early and midterm outcomes of triple patch technique for postinfarction ventricular septal defects. J Thorac Cardiovasc Surg 2016;151:1711-1716.

Pang PY, Sin YK, Lim CH, et al. Outcome and survival analysis of surgical repair of post-infarction ventricular septal rupture. J Cardiothorac Surg 2013;8:44.

Papadopoulos N, Moritz A, Dzemali O et al. Long-term results after surgical repair of postinfarction ventricular septal rupture by infarct exclusion technique. Ann Thorac Surg 2009;87:1421-1425.

Schlotter F, de Waha S, Eitel I, Desch S, Fuernau G, Thiele H. Interventional post-myocardial infarction ventricular septal defect closure: A systematic review of current evidence. EuroIntervention 2016;12:94-102.

Sibal AK, Prasad S, Alison P, Nand P, Haydock D. Acute ischaemic ventricular septal defect-A formidable surgical challenge. Heart Lung Circ 2010;19:71-74.

Statistisches Bundesamt. 2015 Todesursachenstatistik, die 10 häufigsten todesursachen insgesamt. Available at https://www.destatis.de/DE/ ZahlenFakten/GesellschaftStaat/Gesundheit/Todesursachen/Tabellen/ SterbefaelleInsgesamt.html. (Accessed September 2, 2018.)

Takahashi H, Arif R, Almashhoor A, Ruhparwar A, Karck M, Kallenbach $\mathrm{K}$. Long-term results after surgical treatment of postinfarction ventricular septal rupture. Eur J Cardiothorac Surg 2015;47:720-724.

Thiele H, Kaulfersch C, Daehnert I et al. Immediate primary transcatheter closure of postinfarction ventricular septal defects. Eur Heart J 2009;30:81-88.

Trivedi KR, Aldebert P, Riberi A et al. Sequential management of postmyocardial infarction ventricular septal defects. Arch Cardiovasc Dis 2015;108:321-330.

Vondran M, Wehbe M, Leontyev S, et al. Emergency surgery after unsuccessful immediate primary percutaneous post-infarction ventricular septal defect closure: A single center case series. Thorac Cardiovasc Surg 2016;64(suppl 1):OP80.

Yam N, Au TW-K, Cheng L-C. Post-infarction ventricular septal defect: surgical outcomes in the last decade. Asian Cardiovasc Thorac Ann 2013;21:539-545.

Zhu XY, Qin YW, Han YL et al. Long-term efficacy of transcatheter closure of ventricular septal defect in combination with percutaneous coronary intervention in patients with ventricular septal defect complicating acute myocardial infarction: A multicentre study. EuroIntervention 2013;8:1270-1276. 